\title{
Thermoplasmonic Maskless Lithography on Upconverting Nanocomposites Assisted by Gold Nanostars
}

\author{
Eduardo D. Martínez, ${ }^{*,+\uparrow}$ Ricardo R. Urbano, ${ }^{\dagger}$ and Carlos Rettori ${ }^{\dagger, \ddagger}$ \\ † "Gleb Wataghin" Institute of Physics (IFGW), University of Campinas (UNICAMP), \\ 13083-859, Campinas, SP, Brazil. \\ $\ddagger$ Center for Natural and Human Sciences (CCNH), Universidade Federal do ABC \\ (UFABC), 09210-580, Santo André, SP, Brazil. \\ \Present address: INN, Centro Atómico Bariloche, Comisión Nacional de Energía Atómica \\ (CNEA), Consejo Nacional de Investigaciones Científicas y Técnicas (CONICET), Av. E. \\ Bustillo 9500, R8402AGP San Carlos de Bariloche, Río Negro, Argentina \\ *E-mail: edmartin@ifi.unicamp.br
}

\section{Synthesis of UCNPs}

Small size hexagonal core-UCNPs $(<12 \mathrm{~nm})$ were synthesized by the co-precipitation route adapting the protocols reported by Wang and co-workers (Wang et al., 2014) using rare earth acetates as the main precursors. A total of $1 \mathrm{mmol}$ of $\mathrm{X}\left(\mathrm{COOCH}_{3}\right)_{3}(\mathrm{X}$ $=\mathrm{Gd}, \mathrm{Yb}, \mathrm{Er}$ ) was added in a $200 \mathrm{~mL}$ round bottom flask containing $15 \mathrm{~mL}$ of ODE and $9 \mathrm{~mL}$ of OA. The nominal molar ratio of each system was 1:0.78:0.2:0.02 Na:Gd:Yb:Er. The mixture was heated up to $160{ }^{\circ} \mathrm{C}$ for $1 \mathrm{~h}$ to dissolve the precursors and then cooled down to room temperature. At this point, a freshly prepared mixture containing $2.5 \mathrm{~mL}$ of a $1 \mathrm{M} \mathrm{NaOH}$ methanol solution and $10.1 \mathrm{~mL}$ of $0.4 \mathrm{M} \mathrm{NH}_{4} \mathrm{~F}$ solution in methanol was rapidly injected. The flask was heated to $50{ }^{\circ} \mathrm{C}$ for $30 \mathrm{~min}$ and then sealed. The temperature was raised to $100{ }^{\circ} \mathrm{C}$ and the vacuum pump was connected. After $15 \mathrm{~min}$, the vacuum pump was shut down, a condenser was mounted, and the temperature was increased to $280^{\circ} \mathrm{C}$ under constant argon flow. The flask was removed from the mantle 
after $1 \mathrm{~h}$ and allowed to cool down to room temperature. Extraction of the UCNPs was performed by adding excess anhydrous ethanol and centrifugation using $15 \mathrm{~mL}$ Falcon tubes. Centrifugation was performed at $1004 \mathrm{RCF}(2400 \mathrm{rpm})$ for $7 \mathrm{~min}$. The precipitated UCNPs were redispersed in $4 \mathrm{~mL}$ of cyclohexane and ethanol was added to complete the volume.

The centrifugation and washing procedure were repeated twice. Finally, the UCNPs were dispersed in $8 \mathrm{~mL}$ of cyclohexane. As the quantum yield of the UC process is lower for smaller particles due to surface-related quenching processes, a core-shell structure was formed by growing a thin inert layer on the surface of the pre-synthesized core UCNPs. For this, $1 \mathrm{mmol}$ of $\mathrm{Gd}\left(\mathrm{COOCH}_{3}\right)_{3}$ was added to $8 \mathrm{~mL}$ of OA and $12 \mathrm{~mL}$ of ODE in a $200 \mathrm{~mL}$ three neck round bottom flask. The mixture was heated up to 160 ${ }^{\circ} \mathrm{C}$ for $1 \mathrm{~h}$ and then cooled down to room temperature. Then, $6 \mathrm{~mL}$ of the core UCNPs colloid in cyclohexane were added. The subsequent addition of a methanol solution of $\mathrm{NaOH}$ and $\mathrm{NH}_{4} \mathrm{~F}$ and the following procedures were identical to those previously described for the synthesis of core nanoparticles.

\section{Additional analysis of AuNSs}

The amount and sharpness of the AuNS tips have a strong influence on the spectral position of the plasmon absorption band. Figure S1 shows the tips of the AuNS having a typical diameter of $4 \mathrm{~nm}$. This must be considered when analyzing the sintering/melting behavior of the AuNSs layer during the TML process, as discussed in the main text.
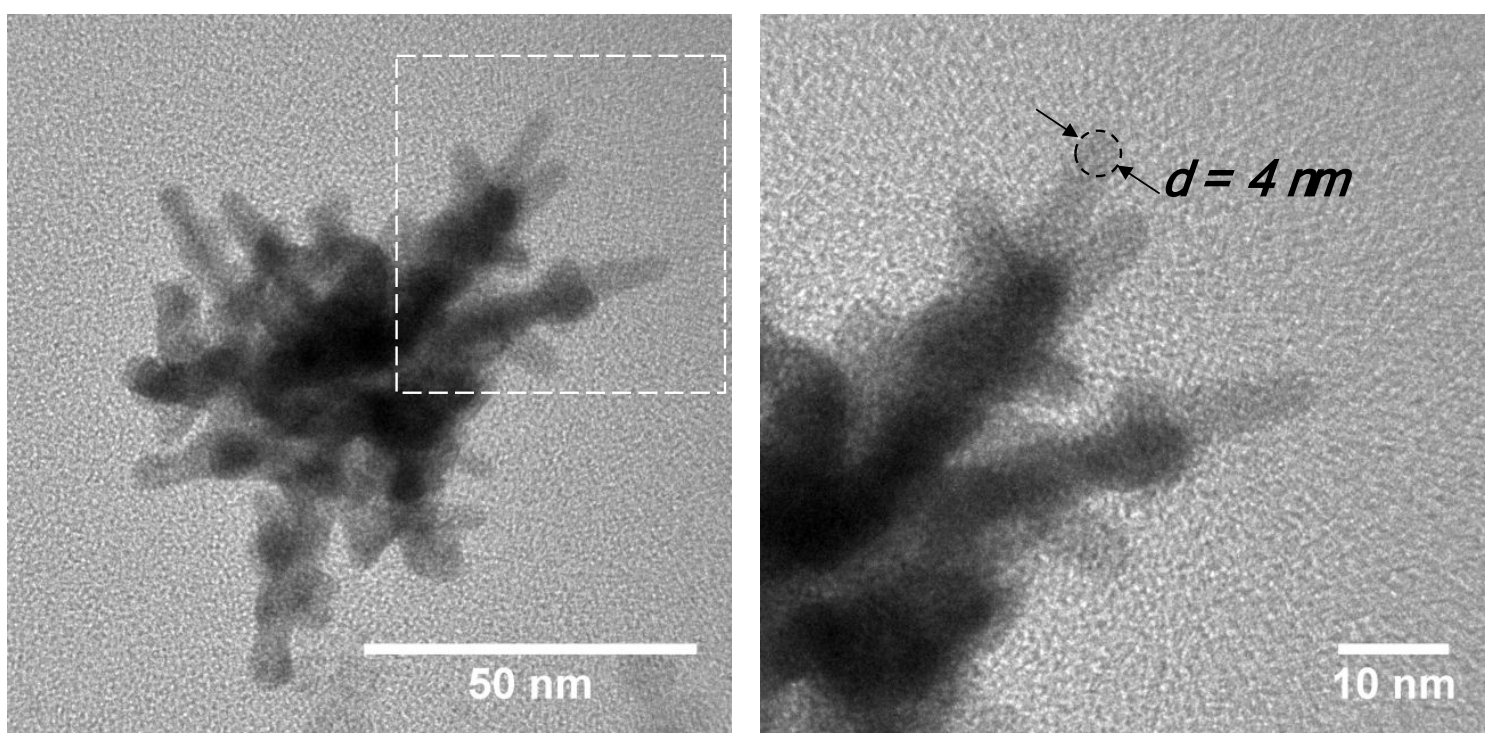

Figure S1. TEM images of an individual AuNS highlighting the fine details of the tips. 


\section{Experimental Set-Up for TML}

The TML process was conducted in a homemade set-up based in an Arduino board and an $\mathrm{x}-\mathrm{y}$ micropositioner (Figure S2). Two stepper motors were coupled to the micrometric screws. The NIR laser beam was focused on the sample mounted on the stage and a servo motor was added to act as a shutter. Patterns were transferred through a GRBL interface connected via USB to the Arduino board. A short video of the writing process is attached to the supporting information.
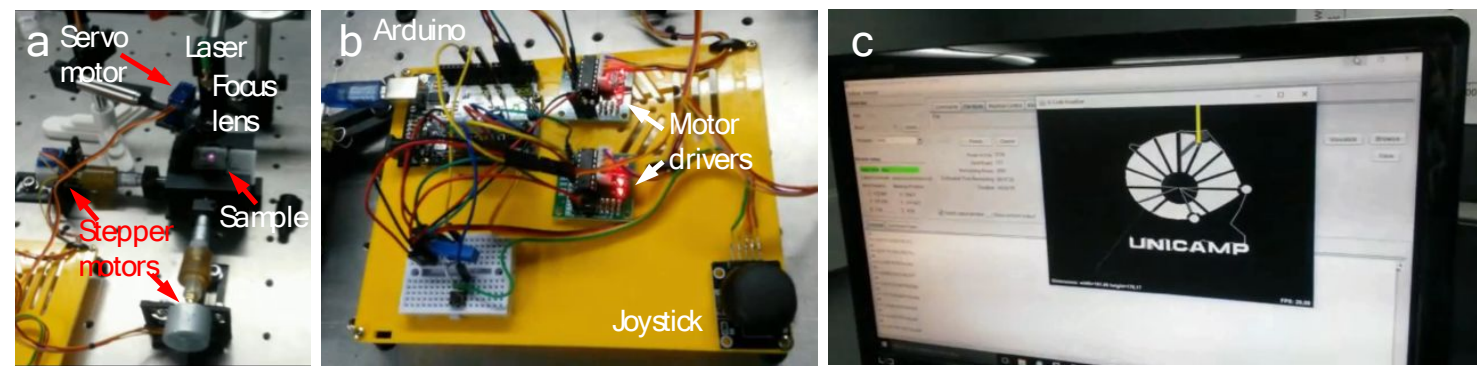

Figure S2. Pictures of the experimental set-up used for the TML. (a) Micropositioner x-y stage coupled to stepper motors. (b) Arduino-based controller. (c) The GRBL interface during the transfer of the UNICAMP logo.

\section{Control of the optical power for TML}

The power density of the NIR excitation laser can be controlled in the TML by adjusting the nominal power in the power source of the laser and measuring the spot size in the position of the sample after focusing the laser beam with a $10 \mathrm{X}$ objective lens. The spot size was determined as the width of the lines imprinted in a sample of the AuNSs/PLA film showed in Figure 4a of the main text. By dividing the nominal power by the area of the spot we obtain the laser power density acting in the sample. The extracted information is presented in Figure S3. In Figure S3a it can be seen a break or disruption in the linear trend of the spot size vs the nominal power. We believe this could be due to a change in the focusing condition at high nominal powers. The divergence of the laser source as a function of the nominal power was not characterized. This same effect can be responsible for the apparent saturation in the laser power density presented in Figure S3b. 

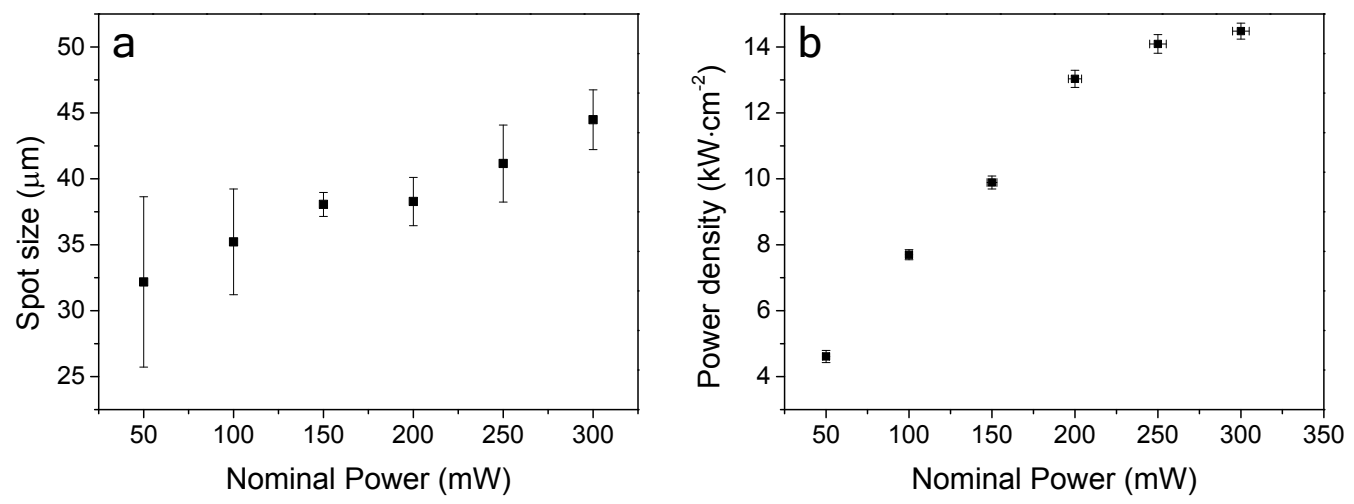

Figure S3. Parameters of the TML process depending on the nominal power of the NIR laser.

(a) Spot size and (b) power density of the laser beam as a function of the nominal power. 